\title{
Solutions of Differential Equations by Evaluations of Functions
}

\author{
By E. Baylis Shanks
}

1. Introduction. The fourth-order formula, known as the Runge-Kutta formula, has been used extensively to obtain approximate solutions of differential equations of first, second, and higher orders. The original idea for such formulas seems to be due to C. Runge [5]. This idea was used more effectively for first-order equations by W. Kutta [3] and for second-order equations by E. J. Nystrom [4]. The extension to order $n$ was made by $\mathrm{R}$. Zurmuhl [6].

The Runge-Kutta fourth-order formula requires only four evaluations of the function $f(x, y)$ involved in the first-order equation $y^{\prime}=f(x, y)$. On the other hand, known formulas of the fifth order, such as the Kutta-Nystrom formula, require six evaluations of the function $f(x, y)$. The sixth-order formulas of A. Huta ([1] and [2]) are not known as well and have the disadvantage of requiring eight evaluations of $f(x, y)$. No formulas of higher order except those developed in this investigation seem to exist in the literature. These formulas are inherently stable, accurate, and "self-starting". Therefore, they are extremely useful either alone or in combination with efficient "continuing" procedures.

Sufficient conditions that a given formula of arbitrary order $m$ be valid are developed in this paper. Necessary and sufficient conditions are given for the validity of a formula of order seven or less. These conditions make it possible to develop more efficient formulas requiring a fewer number of evaluations for orders five and six. Formulas of higher order, including the seventh and eighth orders, are also developed.

2. Preliminary Considerations. The differential equations considered are of the form $y^{\prime}=f(x, y)$ (systems of higher orders may be reduced to systems of the first order). The function $f$ is assumed to be analytic in a sufficiently large neighborhood of the initial point $\left(x_{0}, y_{0}\right)$. Let $f_{i}$ be defined by the equation

$$
f_{i}=f\left(x_{0}+a_{i} h, y_{0}+a_{i} h \sum_{j=0}^{i-1} b_{i j} f_{j}\right) \text {. }
$$

where $f_{0}=f\left(x_{0}, y_{0}\right)$ and $i=1,2, \cdots, n$. Consider the finite series

$$
Y=y_{0}+h \sum_{i=0}^{n} c_{i} f_{i} .
$$

The $a_{i}, b_{i j}, c_{i}$ are parameters to be determined so that $Y$ and the solution $y\left(x_{0}+h\right)$ of the given differential equation will agree to some desired degree of accuracy.

Theorem 1. A necessary and sufficient condition that the Taylor series for $Y$ and the Taylor series for the solution $y\left(x_{0}+h\right)$ of the given differential equation agree through terms in $h^{m}$ is that

$$
\left(f^{(k-1)}\right)_{0}=k \sum_{i=0}^{n} c_{i}\left(f_{i}^{(k-1)}\right)_{0}
$$

Received January 25, 1965. This work has been supported by NASA Contract NAS8-2559, issued at Marshall Space Flight Center, Huntsville, Alabama. 
for $k=1,2, \cdots, m$, where the symbol $f^{(k)}$ is used to denote the kth derivative of the function $f\left(x_{0}+h, y\left(x_{0}+, h\right)\right)$.

The proof is immediate upon comparison of the general terms of the two Taylor series.

In particular, for $k=1$, the condition is

$$
f_{0}=\sum_{i=0}^{n} c_{i} f_{0}
$$

and, for $k=2$, the condition is

$$
\left(f^{\prime}\right)_{0}=2 \sum_{i=0}^{n} c_{i}\left(f_{i}^{\prime}\right)_{0}=2 \sum_{i=0}^{n} c_{i} a_{i}\left[\left(f^{\prime}\right)_{0}+f_{0}\left(f_{y}\right)_{0}\left(\sum_{j=0}^{i-1} b_{i j}-1\right)\right] .
$$

Hence, since $f$ is an arbitrary analytic function, the validity of the conditions for $m \geqq 2$ require

$$
1=\sum_{i=0}^{n} c_{i}=\sum_{j=0}^{i-1} b_{i j}=2 \sum_{j_{1}=1}^{n} c_{j_{1}} a_{j_{1}},
$$

where $i=1,2, \cdots, n$ in $b_{i j}$. It is easily verified that the condition for $k=3$ leads to the requirements

$$
1=3 \sum_{j_{1}=1}^{n} c_{j_{1}} a_{j_{1}}^{2}=6 \sum_{j_{2}=1}^{n} c_{j_{2}} a_{j_{2}} \sum_{j_{1}=1}^{j_{2}-1} b_{j_{2} j_{1}} a_{j_{1}} .
$$

At this point, it is convenient to introduce a "summation convention". It is easily established by an induction that, in each instance, the indices for each $b_{i j}$ are distinct and the second index $j$ is summed for $1,2, \cdots, i-1$, that there are no other changes of indices, and that each $b_{i j}$ is followed immediately by $a_{j}{ }^{k}$ for some positive integer $k$. Hence, without confusion, the summation signs and indices may be omitted, $\sum_{j=1}^{i-1} b_{i j} a_{j}{ }^{k}$ then being denoted by $b a^{k}$, as illustrated next for $k=2$ and $k=3$.

$$
\begin{array}{ll}
k=2: & 1=2 c a \\
k=3: & 1=3 c a^{2} \quad 1=6 c a b a .
\end{array}
$$

In order to further simplify the notation, let $c_{i}^{0}=c_{i}$ and

$$
\sum_{i=j+1}^{n} c_{i}^{(k-1)} a_{i} b_{i j}=c_{j}^{(k)}
$$

for $j=1,2, \cdots, n-k$ and $k=1,2, \cdots, m-2$. When convenient, $c_{i}{ }^{(0)}, c_{i}{ }^{(1)}$, $c_{i}{ }^{(2)}, \cdots$ will be denoted by $c_{i}, c_{i}{ }^{\prime}, c_{i}{ }^{\prime \prime}, \cdots$ respectively.

By straightforward but tedious calculations, the formulas for $k=4,5,6, \cdots$ may be obtained. This was done for $k \leqq 6$ by Huta [1]. The necessary and sufficient conditions for $k \leqq 7$ have been derived by the author and will be given below. The parameters $c_{0}$ and $b_{i 0}$ are determined by two of the equations given in (2) above, which will not be repeated below.

$k=2: \quad 1=2 c a$,

$k=3: \quad 1=3 c a^{2} \quad 1=6 c^{\prime} a$, 


$$
\begin{aligned}
k=4: \quad 1 & =4 c a^{3} \quad 1=12 c^{\prime} a^{2} \quad 1=8 c a^{2} b a \quad 1=24 c^{\prime \prime} a \\
k=5: \quad 1 & =5 c a^{4} \quad 1=20 c^{\prime} a^{3} \quad 1=15 c a^{2} b a^{2} \\
1 & =60 c^{\prime \prime} a^{2} \quad 1=10 c a^{3} b a \quad 1=20 c a^{2}(b a)(b a) \\
7 & =120\left(c a^{2} b a b a+c^{\prime} a^{2} b a\right) \quad 1=120 c^{\prime \prime \prime} a \\
k=6: \quad 1 & =6 c a^{5} \quad 1=30 c^{\prime} a^{4} \quad 1=24 c a^{2} b a^{3} \\
1 & =120 c^{\prime \prime} a^{3} \quad 1=12 c a^{4} b a \quad 1=24 c a^{3}(b a)(b a) \\
1 & =18 c a^{3} b a^{2} \quad 1=48 c a^{2} b a^{2} b a \\
13 & =720 c a^{2}(b a)(b a b a)+360 c^{\prime} a^{2}(b a)(b a) \\
2 & =45\left(c a^{3} b a b a+c^{\prime} a^{3} b a\right) \quad 1=360 c^{\prime \prime \prime} a^{2} \\
1 & =36 c a^{2}(b a)\left(b a^{2}\right) \quad 1=40\left(c a^{2} b a b a^{2}+c^{\prime} a^{2} b a^{2}\right) \\
1 & =720 c^{\prime \prime \prime \prime} a \quad 1=60\left(c a^{2} b a b a b a+c^{\prime} a^{2} b a b a+c^{\prime \prime} a^{2} b a\right) \\
1 & =7 c a^{6} \quad 1=42 c^{\prime} a^{5} \quad 1=35 c a^{2} b a^{4} \\
1 & =210 c^{\prime \prime} a^{4} \quad 1=14 c a^{5} b a \quad 1=21 c a^{4} b a^{2} \\
1 & =28\left(c a^{4} b a b a+c^{\prime} a^{4} b a\right) \quad 1=28 c a^{4}(b a)(b a) \\
1 & =28 c a^{3} b a^{3} \quad 9=280\left(c a^{3} b a^{2} b a+c a^{2} b a^{3} b a\right) \\
5 & =252\left(c a^{3} b a b a^{2}+c^{\prime} a^{3} b a^{2}\right) \quad 1=42 c a^{3}(b a)\left(b a^{2}\right) \\
1 & =105 c a^{2} b a^{2} b a^{2} \quad 31=2520\left(c a^{3} b a b a b a+c^{\prime} a^{3} b a b a+c^{\prime \prime} a^{3} b a\right) \\
5 & =336 c a^{3}(b a)(b a b a)+168 c^{\prime} a^{3}(b a)(b a) \\
19 & =1680\left(c a^{2} b a^{2} b a b a+c a^{2} b a b a^{2} b a+c^{\prime} a^{2} b a^{2} b a\right. \\
11 & =840\left(c a^{2} b a b a^{3}+c^{\prime} a^{2} b a^{3}\right) \\
1 & =80 c a^{2}(b a)\left(b a^{2} b a\right)+40 c a^{2} b a^{2}(b a)(b a) \\
1 & =56 c a^{3}(b a)(b a)(b a) \\
1 & =168\left(c a^{2} b a b a b a^{2}+c^{\prime} a^{2} b a b a^{2}+c^{\prime \prime} a^{2} b a^{2}\right) \\
1 & =63 c a^{2}\left(b a^{2}\right)\left(b a^{2}\right) \\
1 & =280\left(c a^{2} b a b a b a b a+c^{\prime} a^{2} b a b a b a+c^{\prime \prime} a^{2} b a b a+c^{\prime \prime \prime} a^{2} b a\right) \\
1 & =56\left[c a^{2}(b a)\left(b a b a^{2}\right)+c a^{2}\left(b a^{2}\right)(b a b a)+c^{\prime} a^{2}(b a)\left(b a^{2}\right)\right] \\
1 & =56 c a^{2}(b a)\left(b a^{3}\right) \quad 1=840 c^{\prime \prime \prime} a^{3} \quad 1=2520 c^{\prime \prime \prime \prime} a^{2} \\
1 & =5040 c^{\prime \prime \prime \prime \prime} a \\
19 & =1260\left[2 c a^{2}(b a)(b a b a b a)+c a^{2}(b a b a)^{2}+2 c^{\prime} a^{2}(b a)(b a b a)+c^{\prime \prime} a^{2}(b a)^{2}\right]
\end{aligned}
$$

This list could be extended, although the computation would be laborious. Instead, sufficient conditions will be developed which are general and relatively easy to express in a list of formulas. 
3. Sufficient Conditions. Let

$$
f_{00}=f(x, y), \quad f_{i j}=\frac{\delta^{i+j} f}{\delta x^{i} \delta y^{j}},
$$

where $x$ is a linear function of $h$ and $y$ is an analytic function of $h$. As before, assume that $f(x, y)$ is an arbitrary analytic function. Also, let

$$
(n, j)=\sum_{k=0}^{n}\left(\begin{array}{l}
n \\
k
\end{array}\right) x^{\prime n-k} y^{\prime k} f_{n-k, j+k}
$$

The following theorem is well known and easy to prove.

THEOREM 2. If primes denote differentiation with respect to $h$ and the notation in (4) is used, then

$$
(n, j)^{\prime}=(n+1, j)+n y^{\prime \prime}(n-1, j+1) .
$$

The following theorem will be proved.

Theorem 3. If $n=i_{2}+i_{3}+\cdots+i_{k}, k=i_{1}+2 i_{2}+\cdots+k i_{k}$, and $C\left(i_{1}, i_{2}, \cdots, i_{k}\right)$ is a constant depending upon $i_{1}, i_{2}, \cdots, i_{k}$, then $f^{(k)}$ can be expressed in the following form

$$
f^{(k)}=\sum C\left(i_{1}, i_{2}, \cdots, i_{k}\right) y^{\prime \prime i_{2}} \cdots\left(y^{(k)}\right)^{i_{k}}\left(i_{1}, n\right) .
$$

Proof. The proof is made by use of complete induction. For $k=1, f^{\prime}=(1,0)$, $C(1)=1$, and $n=0$ so that the theorem is true in this case.

Assume the theorem is true for $k$. Then, after differentiation of $f^{(k)}$, the terms of $f^{(k+1)}$ can be classified into the following types.

$$
\begin{gathered}
i_{j} C\left(i_{1}, i_{2}, \cdots, i_{k}\right) y^{\prime \prime i_{2}} \cdots\left(y^{(j-1)}\right)^{i_{j-1}}\left(y^{(j)}\right)^{i_{j}-1}\left(y^{(j+1)}\right)^{i_{j+1}+1} \cdots\left(y^{(k)}\right)^{i_{k}}\left(i_{1, n}\right), \\
C\left(i_{1}, i_{2}, \cdots, i_{k}\right) y^{\prime \prime i_{2}} \cdots\left(y^{k}\right)^{i_{k}}\left[\left(i_{1}+1, n\right)+i_{1} y^{\prime \prime}\left(i_{1}-1, n+1\right)\right],
\end{gathered}
$$

where $i_{k+1}=0$ when $j=k$ in the first type. It is readily verified that $k+1=$ $i_{1}^{\prime}+2 i_{2}^{\prime}+\cdots+(k+1) i_{k+1}^{\prime}$ for each type while $n=i_{2}{ }^{\prime}+\cdots+i_{k+1}^{\prime}$ for the first type and $n+1=i_{2}{ }^{\prime}+\cdots+i_{k+1}^{\prime}$ for the second type. Hence the theorem is true for $k+1$ and the induction is complete.

The results established by Theorems 2 and 3 may be applied to the function $f(x, y)$, where $y^{\prime}=f(x, y), x=x_{0}+h, y=y\left(x_{0}+h\right)$, and $y_{0}=y\left(x_{0}\right)$ or to the function $f_{i}=f\left(x_{i}, y_{i}\right)$, where $Y=y_{0}+h \sum_{i=0}^{n} c_{i} f_{i}, x_{i}=x_{0}+a_{i} h$, and $y_{i}=y_{0}+a_{i} h \sum_{j=0}^{i-1} b_{i j} f_{j}$.

Theorem 4. For the function $f(x, y), f^{(k)}=\sum_{t} \pi_{t}{ }^{[k]}$, where $\pi_{t}{ }^{[k]}$ is a product of an integer and factors of the type $(n, j)$ for $k \geqq 1$.

The proof is immediate, using complete induction, since $y^{\prime \prime}=f^{\prime}=(1,0)$ and $(n, j)^{\prime}=(n+1, j)+n(1,0)(n-1, j+1)$.

The next theorem gives an expression for $f_{j}^{(k)}$ at the initial point $\left(x_{0}, y_{0}\right)$. In order to state the theorem more concisely, let us define $Q_{i 1}^{[1]}=a_{i}$ and, inductively,

$$
Q_{i t}^{[k]}=a_{i}^{i_{1}}\left(\prod_{r=1}^{i_{2}} 2 a_{i} \sum_{j=1}^{i-1} b_{i j} Q_{j t_{r}}^{[1]}\right) \cdots\left(\prod_{r=1}^{i_{k}} k a_{i} \sum_{j=1}^{i-1} b_{i j} Q_{j t_{r}}^{[k-1]}\right),
$$

where the induction is on $k, k=i_{1}+2 i_{2}+\cdots+k i_{k}$, and the subscripts $t, t_{r}$ are used simply to number the distinct terms defined for each fixed $k$, the order of the 
numbering being immaterial. For example, when $k=2$ the possible values for $i_{1}$ and $i_{2}$ are $i_{1}=2, i_{2}=0$ or $i_{1}=0, i_{2}=1$ so that $t=1,2$ and we may write

$$
Q_{i 1}^{[2]}=a_{i}^{2}, \quad Q_{i 2}^{[2]}=2 a_{i} \sum_{j=1}^{i-1} b_{i j} a_{j} .
$$

THEOREM 5. If $f^{(k)}=\sum_{t} \pi_{t}{ }^{[k]}$, where $\pi_{t}{ }^{[k]}$ is a product of an integer and factors of the type $(n, j)$ and $k \geqq 1$, then at $\left(x_{0}, y_{0}\right)$

$$
f_{j}^{(k)}=\sum_{t} Q_{j i}^{[k]} \pi_{t}^{[k]}
$$

for a suitable numbering of the $Q_{j t}^{[k]}$.

Proof. The theorem is proved if it is shown that the quotient of corresponding typical terms of $f_{j}^{(k)}$ and $f^{(k)}$ is $Q_{j t}^{[k]}$ for each value of the index $t$. The proof is made by use of complete induction on $k$.

For $k=1, f^{\prime}=\left(1_{\diamond}, 0\right)$ and $\left(f_{j}^{\prime}\right)_{0}=a_{j}(1,0)$ so that $Q_{j t}^{[1]}=a_{j}$ and $i_{1}=1$, which establishes the theorem in this case.

Assume the theorem is true for $1,2, \cdots, k-1$ and consider $f^{(k)}$ and $f_{j}^{(k)}$. It follows from Theorem 3 that

$$
\begin{aligned}
f^{(k)} & =\sum C\left(i_{1}, i_{2}, \cdots, i_{k}\right) y^{\prime \prime i_{2}} \cdots\left(y^{(k)}\right)^{i_{k}}\left(i_{1}, n\right), \\
f_{i}^{(k)} & =\sum C\left(i_{1}, i_{2}, \cdots, i_{k}\right) y_{i}{ }^{\prime \prime i_{2}} \cdots\left(y_{i}{ }^{(k)}\right)^{i_{k}}\left(i_{1}, n\right)_{i} .
\end{aligned}
$$

It is also true by Theorem 4 that

and at $\left(x_{0}, y_{0}\right)$

$$
y^{(r)}=f^{(r-1)}=\sum_{t} \pi_{t}{ }^{[r-1]}
$$

$$
y_{i}^{(r)}=r a_{i} \sum_{j} b_{i j} \sum_{t} Q_{j t}^{[r-1]} \pi_{t}^{[r-1]} .
$$

The latter equation is equivalent to

$$
y_{i}^{(r)}=\sum_{t}\left[r a_{i} \sum_{j} b_{i j} Q_{j t}^{[r-1]}\right] \pi_{t}^{[r-1]} .
$$

A typical term of $f^{(k)}$ is obtained by fixing $i_{1}, i_{2}, \cdots, i_{k}$ in equation (6) and then fixing $t$ in equation (8) for each value of $r$ represented in the term from equation (6). The corresponding typical term of $f_{i}^{(k)}$ is obtained by fixing the indices in the same way in equations (7) and (9). Since $\left(i_{1}, n\right)_{i}=a_{i}^{i_{1}}\left(i_{1}, n\right)$, it follows that the quotient of corresponding typical terms from $f_{i}^{(k)}$ and $f^{(k)}$ is precisely $Q_{i t}^{[k]}$ as given in the theorem. Hence the theorem is true.

A necessary and sufficient condition that the Taylor series for $Y$ and $y\left(x_{0}+h\right)$ agree through terms in $h^{m}(m \geqq 2)$ is that

$$
1=\sum_{j=0}^{n} c_{j}, \quad 1=\sum_{j=0}^{i-1} b_{i j}
$$

for $i=1,2, \cdots, n$ and

$$
\sum_{t} \pi_{t}{ }^{[k]}=(k+1) \sum_{i} c_{i} \sum_{t} Q_{i t}^{[k]} \pi_{t}^{[k]}
$$

for $k=1,2, \cdots, m-1$. This follows immediately from Theorems 1 and 5 and is incorporated in the following theorem. 
Theorem 6. A necessary and sufficient condition that the Taylor series for $Y$ and the Taylor series for $y\left(x_{0}+h\right)$ agree through terms in $h^{m}(m \geqq 2)$ is that

$$
\sum_{t}\left[1-(k+1) \sum_{i} c_{i} Q_{i t}^{[k]}\right] \pi_{t}^{[k]}=0
$$

for $k=1,2, \cdots, m-1$ and

$$
1=\sum_{j=0}^{n} c_{j}=\sum_{j=0}^{i-1} b_{i j}
$$

for $i=1,2, \cdots, n$.

Sufficient conditions are implied by Theorem 6 and given in the next theorem.

Theorem 7. Sufficient conditions for the Taylor series for $Y$ and the Taylor series for $y\left(x_{0}+h\right)$ to agree through terms in $h^{m}(m \geqq 2)$ are

$$
1=\sum_{j=0}^{n} c_{j}=\sum_{j=0}^{i-1} b_{i j}
$$

for $i=1,2, \cdots, n$ and

$$
1=(k+1) \sum_{i=1}^{n} c_{i} Q_{i t}^{[k]}
$$

for $k=1,2, \cdots, m-1$ and $t \geqq 1$.

The equations (10) state the conditions to be satisfied for formulas of different orders. These equations are completely determined when the $Q_{i t}^{[k]}$ are known. Due to this importance of these quantities, we shall elaborate further on their formation.

Observe that, in (5), $a_{i}$ is multiplied by $1,2 a_{i} b_{i j}$ is multiplied and summed on $j$ by a term $Q_{j t_{r}}^{[1]}, \cdots$, and $k a_{i} b_{i j}$ is multiplied and summed on $j$ by a term $Q_{j t_{s}}^{[k-1]}$. Hence the equation (5) is a rule that states that a term $Q_{i t}^{[k]}$ is made by forming a product consisting of $i_{1}$ factors of the type mentioned first in the preceding sentence, $i_{2}$ factors of the type mentioned second in the preceding sentence, $\cdots$, and $i_{k}$ factors of the type mentioned last in the preceding sentence, where $i_{1}+2 i_{2}+\cdots+k i_{k}=$ $k$. Since $Q_{i t}^{[1]}=a_{i}$ and $t=1$ in this case, the succeeding $Q_{i t}^{[k]}$ may be formed inductively.

For example, when $k=3$ the possible values of $i_{1}, i_{2}, i_{3}$ are $i_{1}=3, i_{2}=i_{3}=$ $0 ; i_{1}=1, i_{2}=1, i_{3}=0 ; i_{1}=i_{2}=0, i_{3}=1$. There are two terms for $i_{3}=1$ since $Q_{j t_{r}}^{[2]}$ is involved. Hence the rule leads to the following four terms

$$
\begin{aligned}
& Q_{i 1}^{[3]}=a_{i}^{3}, \quad Q_{i 2}^{[3]}=a_{i}\left(2 a_{i} \sum b_{i j} a_{j}\right)=2 a_{i}{ }^{2} \sum b_{i j} a_{j}, \\
& Q_{i 3}^{[3]}=3 a_{i} \sum b_{i j} a_{j}{ }^{2}, \quad Q_{i 4}^{[3]}=3 a_{i} \sum_{j=1}^{i-1} b_{i j} 2 a_{j} \sum_{k=1}^{j-1} b_{j k} a_{k} .
\end{aligned}
$$

Note that $i$ is a free index, which is to be summed when $Q_{i t}^{[k]}$ is inserted in equation (10), while $t$ or $t_{r}$ number the terms of a given type.

These results can be expressed in the "summation convention" notation. To illustrate this fact and to recapitulate previous results, a list is given below (in the summation convention) of the $Q^{[k]}$ for $k=1,2,3,4$. Formation of the list is made easier by grouping terms of the type $p a b Q^{[p-1]}$ separately as shown. 


$$
\begin{aligned}
& k=1: \quad a \\
& k=2: a^{2} \quad 2 a b a \\
& k=3: a^{3} \quad 2 a^{2} b a \quad 3 a b a^{2} \quad 6 a b a b a \\
& k=4: \quad a^{4} \quad 2 a^{3} b a \quad 3 a^{2} b a^{2} \quad 4 a b a^{3} \quad 8 a b a^{2} b a \\
& 6 a^{2} b a b a \quad 4 a^{2}(b a)(b a) \quad 12 a b a b a^{2} \quad 24 a b a b a b a
\end{aligned}
$$

This list can be extended by the rule with relative ease.

It is possible to state a rule so that the equations for a given $k$ can be written without reference to the equations for smaller integers. Such a rule follows essentially from the following facts, which are consequences of equations (5) and (10): (1) $Q^{[k]}$ is a product of factors, each factor of which consists of a sequence of an integer, $a$ 's, and $b a^{k,} \mathrm{~s},(2)$ in each factor of $Q^{[k]}$, at least one $a$ immediately precedes each $b_{i j}$ and at least one $a$ immediately follows each $b_{i j},(3)$ in each factor of $Q^{[k]}$, the integer $p$ immediately precedes $b a^{k}$ if and only if $p-1$ is the number of $a$ 's that follow and are connected by summation to the given $b_{i j}$, and (4) $Q^{[k]}$ contains exactly the number $k$ of $a$ 's. However, such a rule is not of much advantage since all equations for $k=1,2, \cdots, m-1$ are part of the sufficient conditions to be satisfied in order to obtain a valid formula of order $m$.

To illustrate the remarks just made, consider the case when $k=5$. First, one would form a sequence of $a$ 's and $b a^{k}$ 's according to the requirements in (1), (2), and (4). One such sequence would be $a^{2} b a^{2} b a$ so that the term under consideration would be $c a^{2} b a^{2} b a$. According to the requirement (3), integers should appear in the final expression, giving (6) $c a^{2}(4) b a^{2}(2) b a$. Then, according to equation (10), the final equation of condition would be

$$
1=48 c a^{2} b a^{2} b a \text {. }
$$

It should be observed that the values of $k$ in the latter discussion do not correspond to those in Section 2 .

4. Specification of a Formula. In order to specify a given formula of order $m$ with $n+1$ evaluations, denoted $(m, n+1)$, one may detach the coefficients as follows:

$$
\begin{array}{ll} 
& \text { Formula }(m, n+1) \\
a_{1} & a_{1} b_{10} \\
a_{2} & a_{2} b_{20}+a_{2} b_{21} \\
\vdots & \vdots \\
a_{n} & a_{n} b_{n 0}+a_{n} b_{n 1}+\cdots+a_{n} b_{n, n-1} \\
& c_{0}+c_{1}+\cdots+c_{n} .
\end{array}
$$

This array specifies the formula

$$
Y=y_{0}+h \sum_{i=0}^{n} c_{i} f_{i}
$$


where

$$
\begin{aligned}
f_{0}= & f\left(x_{0}, y_{0}\right) \\
f_{1}= & f\left(x_{0}+a_{1} h, y_{0}+a_{1} h b_{10} f_{0}\right) \\
f_{2}= & f\left(x_{0}+a_{2} h, y_{0}+a_{2} h \sum_{j=0}^{1} b_{2 j} f_{j}\right) \\
& \vdots \\
f_{n}= & f\left(x_{0}+a_{n} h, y_{0}+a_{n} h \sum_{j=0}^{n-1} b_{n j} f_{j}\right) .
\end{aligned}
$$

Such arrays will be used below to specify various formulas.

5. General Formulas for Orders Below 5. In these cases, it is possible to give formulas with $n$ equal to its minimum value $m-1$.

The formulas for $m \leqq 3$ are easy to derive and are simply listed next.

$$
\begin{array}{r}
m=0: \quad Y=y_{0} \\
m=1: \quad Y=y_{0}+h f_{0} \\
m=2: \quad a_{1} \quad a_{1} \\
\frac{1}{2 a_{1}}\left[\left(2 a_{1}-1\right)+1\right], \\
m=3: \quad \text { I. } \quad \frac{2}{3} \quad \frac{2}{3} \\
\quad \frac{2}{3} \frac{1}{4 c} \\
\frac{1}{4}[1+(3-6 c)+6 c], \\
\text { II. } \quad a_{1} \quad a_{1} \\
a_{2} a_{2}\left(b_{20}+b_{21}\right) \\
\left(c_{0}+c_{1}+c_{2}\right),
\end{array}
$$

where

$$
\begin{aligned}
b_{21} & =\frac{\left(a_{2}-a_{1}\right)}{a_{1}\left(2-3 a_{1}\right)}, & b_{20} & =1-b_{21}, \\
c_{1} & =\frac{2-3 a_{2}}{6 a_{1}\left(a_{1}-a_{2}\right)}, & c_{2} & =\frac{2-3 a_{1}}{6 a_{2}\left(a_{2}-a_{1}\right)}, \quad c_{0}=1-c_{1}-c_{2} .
\end{aligned}
$$

The last three formulas have been given with detached coefficients as explained previously.

For order 4 with $n=3$, let

$$
\vec{b}_{i}=\sum_{j=1}^{i-1} b_{i j} a_{j}-\frac{1}{2} a_{i}
$$

for $i=1,2,3$ where $\bar{b}_{1}=-a_{1} / 2$. Then three of the seven conditions may be written

$$
c a \bar{b}=0, \quad c a^{2} \bar{b}=0, \quad c^{\prime} a \bar{b}=0 .
$$


As vectors in three space, $c, c a$, and $c^{\prime}$ are orthogonal to the nonzero vector $a \bar{b}$ and hence are linearly dependent. Thus, for $i=1,2,3$

$$
A c_{i}+B c_{i} a_{i}+C c_{i}{ }^{\prime}=0
$$

for some nonzero vector $(A, B, C)$. Multiplying and summing in turn by $a_{i}$ and $a_{i}^{2}$, we get

$$
\begin{aligned}
& \frac{1}{2} A+\frac{1}{3} B+\frac{1}{6} C=0, \\
& \frac{1}{3} A+\frac{1}{4} B+\frac{1}{12} C=0 .
\end{aligned}
$$

These equations imply, for each $i, c_{i}-c_{i} a_{i}=c_{i}^{\prime}$ and $a_{3}=1$ since $c_{3} \neq 0$. With this information, it is not difficult to derive the complete solution for order 4 .

First, $a_{2} \neq 1$. Otherwise, $c_{2}^{\prime}=0$ and $c_{1}^{\prime \prime}=c_{2}^{\prime} a_{2} b_{21}=0$, but this contradicts the condition $1=24 c_{1}^{\prime \prime} a_{1}$. This leaves three cases, which are discussed below.

Case 1. $a_{1}=a_{2}$. Then $1=6\left(c_{1}^{\prime}+c_{2}^{\prime}\right) a_{2}$ and $1=12\left(c_{1}^{\prime}+c_{2}^{\prime}\right) a_{2}^{2}$, which implies $a_{1}=a_{2}=\frac{1}{2}$ and $c_{1}^{\prime}+c_{2}^{\prime}=\frac{1}{3}$. Let $c_{2}=2 c$. Then the formula for this case is

$$
\begin{array}{ll}
\frac{1}{2} & \frac{1}{2} \\
\frac{1}{2} & \frac{1}{12 c}[(6 c-1)+1] \\
& 1 \quad[0+(1-6 c)+6 c] \\
& \frac{1}{6}[1+(4-12 c)+12 c+1] .
\end{array}
$$

This formula for $1=6 c$ is the famous Runge-Kutta Fourth Order Formula.

Case 2. $a_{1}=1$. Then $c_{1}{ }^{\prime}=0,6 c_{2}{ }^{\prime} a_{2}=1$, and $12 c_{2}{ }^{\prime} a_{2}{ }^{2}=1$, which implies that $a_{2}=\frac{1}{2}$ and $c_{2}^{\prime}=\frac{1}{3}$. Then $c_{2}=c_{2}^{\prime} /\left(1-a_{2}\right)=\frac{2}{3}$, so that $c_{1}+c_{3}=\frac{1}{2}-c_{2} a_{2}=$ $\frac{1}{2}-\frac{1}{3}=\frac{1}{6}$. Let $f_{3}=c$. Then the formula for this case is

$$
\begin{array}{ll}
1 & 1 \\
\frac{1}{2} & \frac{1}{8}(3+1) \\
1 & \frac{1}{12 c}[(12 c-3)+(-1)+4] \\
& \frac{1}{6}[1+(1-6 c)+4+6 c] .
\end{array}
$$

Case 3. $a_{1}, a_{2}, 1$ are distinct. Then

$$
c_{1}^{\prime \prime}=\frac{1}{24 a_{1}}, \quad c_{1}^{\prime}=\frac{1-2 a_{2}}{12 a_{1}\left(a_{1}-a_{2}\right)}, \quad c_{2}^{\prime}=\frac{1-2 a_{1}}{12 a_{2}\left(a_{2}-a_{1}\right)} .
$$

The formula for this case is

$$
\begin{array}{ll}
a_{1} & a_{1} \\
a_{2} & a_{2}\left(b_{20}+b_{21}\right) \\
1 & \left(b_{30}+b_{31}+b_{32}\right) \\
\left(c_{0}+c_{1}+c_{2}+c_{3}\right),
\end{array}
$$


where

$$
\begin{aligned}
b_{21} & =\frac{a_{2}-a_{1}}{2 a_{1}\left(1-2 a_{1}\right)}, \quad b_{20}=\frac{3 a_{1}-4 a_{1}{ }^{2}-a_{2}}{2 a_{1}\left(1-2 a_{1}\right)}, \\
b_{31} & =\frac{\left(1-a_{1}\right)\left(2-a_{1}-5 a_{2}+4 a_{2}^{2}\right)}{2 a_{1}\left(a_{1}-a_{2}\right)\left(3-4 a_{1}-4 a_{2}+6 a_{1} a_{2}\right)}, \\
b_{32} & =\frac{\left(1-a_{1}\right)\left(1-a_{2}\right)\left(1-2 a_{1}\right)}{a_{2}\left(a_{2}-a_{1}\right)\left(3-4 a_{1}-4 a_{2}+6 a_{1} a_{2}\right)}, \\
b_{30} & =1-b_{31}-b_{32}, \\
c_{1}= & \frac{2 a_{2}-1}{12 a_{1}\left(a_{1}-a_{2}\right)\left(a_{1}-1\right)}, \quad c_{2}=\frac{2 a_{1}-1}{12 a_{2}\left(a_{2}-a_{1}\right)\left(a_{2}-1\right)} \\
c_{3}= & \frac{3-4 a_{1}-4 a_{2}+6 a_{1} a_{2}}{12\left(a_{1}-1\right)\left(a_{2}-1\right)}, \quad c_{0}=1-c_{1}-c_{2}-c_{3} .
\end{aligned}
$$

6. Inconsistency of Conditions when $m=n+1=5$. It will now be shown that the fifteen conditions for order 5 are inconsistent when $n=4$, the minimum value. Let

$$
\bar{b}_{i}=\sum_{j=1}^{i-1} b_{i j} a_{j}-\frac{1}{2} a_{i}
$$

for $i=1,2,3,4$ where $\bar{b}_{1}=-a_{1} / 2$. Then six of the conditions may be written

$$
c a \bar{b}=c a^{2} \bar{b}=c a^{3} \bar{b}=c^{\prime} a \bar{b}=c^{\prime \prime} a \bar{b}=\left(c a^{2} b+c^{\prime} a^{2}\right) \bar{b}=0 \text {. }
$$

In four space, the vectors $c, c a, c a^{2}, c^{\prime}, c^{\prime \prime}$, and $\sum_{i=j+1}^{n} c_{i} a_{i}{ }^{2} b_{i j}+c_{j}^{\prime} a_{j}$ for $j=1$, $2,3,4$ are orthogonal to the nonzero vector $a \bar{b}$. Since $c_{4}^{\prime}=c_{4}^{\prime \prime}=c_{3}^{\prime \prime}=0$ by convention,

$$
A c_{j}+B c_{j}{ }^{\prime}+C c_{j}^{\prime \prime}=0
$$

implies $A=B=C=0$. Hence $c, c^{\prime}$, and $c^{\prime \prime}$ are independent vectors, spanning the three space orthogonal to the space spanned by the vector $a \bar{b}$. By a straightforward calculation, we get

$$
\begin{aligned}
c a= & a_{4} c+\left(7-8 a_{4}\right) c^{\prime}-20\left(1-a_{4}\right) c^{\prime \prime}, \\
c a^{2}= & a_{4}{ }^{2} c+\left(6-8 a_{4}{ }^{2}\right) c^{\prime}-2\left(9-10 a_{4}{ }^{2}\right) c^{\prime \prime}, \\
& \sum_{i=j+1}^{n} c_{i} a_{i}{ }^{2} b_{i j}+c_{j}{ }^{\prime} a_{j}=2 c_{j}{ }^{\prime}-3 c_{j}^{\prime \prime}
\end{aligned}
$$

for $j=1,2,3,4$. From the last of these equations $c_{4} a_{4}{ }^{2} b_{43}+c_{3}{ }^{\prime} a_{3}=2 c_{3}{ }^{\prime}$, which, since $c_{4} a_{4} b_{43}=c_{3}^{\prime} \neq 0$, is equivalent to $a_{3}+a_{4}=2$. From the first two equations

$$
\begin{aligned}
c_{3}\left(a_{3}-a_{4}\right) & =\left(7-8 a_{4}\right) c_{3}{ }^{\prime}, \\
c_{3}\left(a_{3}{ }^{2}-a_{4}{ }^{2}\right) & =\left(6-8 a_{4}{ }^{2}\right) c_{3}{ }^{\prime},
\end{aligned}
$$

from which it follows that

$$
\left(a_{3}+a_{4}\right)\left(7-8 a_{4}\right) c_{3}{ }^{\prime}=\left(6-8 a_{4}{ }^{2}\right) c_{3}{ }^{\prime} .
$$


Since $c_{3}{ }^{\prime} \neq 0$ and $a_{3}+a_{4}=2$, it follows that $a_{4}=1$ and hence $a_{3}=1$ also. Since $c_{3}-c_{3} a_{3}=c_{3}^{\prime}$, we get $c_{3}{ }^{\prime}=0$, a contradiction.

This contradiction establishes the claim that all of the conditions cannot be satisfied when $m=n+1=5$.

7. A Change in Viewpoint. One procedure, which was adopted by Kutta, is to increase $n$ from 4 to 5 . This introduces additional parameters, which enabled Kutta to formulate a formula of the fifth order with six evaluations. His formula contained an error, which was later corrected by Nystrom. However, it is not necessary to increase $n$ from 4 to 5 if we adopt a new viewpoint.

In the general case, let us require only that the conditions be approximately satisfied. In other words, if a condition is written in the form $F=0$, then the new requirement is to be that $F=e$ for a sufficiently small $e$. In particular, for a given order $m$, the possibility will be examined that all conditions whose orders are below $m$ are satisfied precisely, while some or all of order $m$ are approximately satisfied.

8. Formulas with Five Evaluations. For $m=5$, let $a_{4}=1, c_{i}-c_{i} a_{i}=c_{i}{ }^{\prime}$ for $i=1,2,3,4$, and $\bar{b}_{i}=0$ for $i=2,3,4$. Let it be required that the conditions whose orders are below five be satisfied. Then $1=6 c^{\prime} a=6 c a b=3 c a^{2}-3 c_{1} a_{1}^{2}$, so that $c_{1}=0$ since $1=3 c a^{2}$. Thus $c_{1}^{\prime}=0$ and the new requirements reduce to the following

$$
\begin{aligned}
& 1=6 c^{\prime} a, \quad 1=12 c^{\prime} a^{2}, \quad 1-20 c^{\prime} a^{3}=e^{\prime}, \\
& 1=2 c a, \quad 1=24 c^{\prime \prime} a, \quad 1-60 c^{\prime \prime} a^{2}=e^{\prime \prime}, \\
& 1-120 c^{\prime \prime \prime} a=e^{\prime \prime \prime},
\end{aligned}
$$

where $e^{\prime}, e^{\prime \prime}$, and $e^{\prime \prime \prime}$ are to be taken sufficiently small. Since $c_{1}{ }^{\prime}=0$,

$$
3 e^{\prime}=3-5 a_{2}-5 a_{3}+10 a_{2} a_{3}
$$

and, since $a_{2}=2 b_{21} a_{1}=2 c_{1}^{\prime \prime \prime} a_{1} / c_{2}^{\prime \prime} a_{2}$, it follows that

$$
a_{1}\left(2-5 a_{2}\right)=2 a_{2} e^{\prime \prime}+2 e^{\prime \prime \prime}\left(a_{1}-a_{2}\right) .
$$

To recapitulate, there are six parameters $e^{\prime}, e^{\prime \prime}, e^{\prime \prime \prime}, a_{1}, a_{2}, a_{3}$ and five requirements, namely, $e^{\prime}, e^{\prime \prime}, e^{\prime \prime \prime}$ are to be taken sufficiently small while (11) and (12) are to hold. When these requirements are met, a valid formula is obtained as given by the array below.

$$
\begin{array}{ll}
a_{1} & a_{1} \\
a_{2} & a_{2}\left(b_{20}+b_{21}\right) \\
a_{3} & a_{3}\left(b_{30}+b_{31}+b_{32}\right) \\
1 & \left(b_{40}+b_{41}+b_{42}+b_{43}\right) \\
& \left(c_{0}+c_{1}+c_{2}+c_{3}+c_{4}\right),
\end{array}
$$

where

$$
\sum_{i=j+1}^{4} c_{i}^{(k)} a_{i} b_{i j}=c_{j}^{(k+1)}
$$


for $j=1,2, \cdots, 3-k$ and $k=0,1,2$;

$$
b_{i 0}=1-b_{i 1}-\cdots-b_{i, i-1}
$$

for $i=2,3,4$; and

$$
\begin{aligned}
& c_{1}^{\prime \prime \prime}=\frac{1-e^{\prime \prime \prime}}{120 a_{1}}, \quad c_{1}^{\prime \prime}=\frac{-2 e^{\prime \prime}+2-5 a_{2}}{120 a_{1}\left(a_{1}-a_{2}\right)}, \quad c_{2}^{\prime \prime}=\frac{-2 e^{\prime \prime}+2-5 a_{1}}{120 a_{2}\left(a_{2}-a_{1}\right)}, \\
& c_{1}^{\prime}=0, \quad c_{2}^{\prime}=\frac{1-2 a_{3}}{12 a_{2}\left(a_{2}-a_{3}\right)}, \quad c_{3}^{\prime}=\frac{1-2 a_{2}}{12 a_{3}\left(a_{3}-a_{2}\right)}, \\
& c_{1}=0, \quad c_{2}=\frac{1-2 a_{3}}{12 a_{2}\left(1-a_{2}\right)\left(a_{2}-a_{3}\right)}, \quad c_{4}=\frac{1}{2}-c_{2} a_{2}-c_{3} a_{3}, \\
& c_{3}=\frac{1-2 a_{2}}{12 a_{3}\left(1-a_{3}\right)\left(a_{3}-a_{2}\right)}, \quad c_{0}=1-c_{1}-c_{2}-c_{3}-c_{4}, \\
& 3 e^{\prime}=3-5\left(a_{2}+a_{3}\right)+10 a_{2} a_{3}, \\
& a_{1}\left(2-5 a_{2}\right)=2 a_{2} e^{\prime \prime}+2 e^{\prime \prime \prime}\left(a_{1}-a_{2}\right), \\
& e^{\prime}, e^{\prime \prime}, e^{\prime \prime \prime} \text { chosen sufficiently small. }
\end{aligned}
$$

It is clear that the requirements can be met and that the array defines a four parameter set of valid formulas, each of which involves only five evaluations of the function.

According to the traditional viewpoint, all of these formulas would be called fourth-order formulas since $e^{\prime}, e^{\prime \prime}$, and $e^{\prime \prime \prime}$ cannot be simultaneously zero. However, when $e^{\prime}, e^{\prime \prime}$, and $e^{\prime \prime \prime}$ are chosen sufficiently small, such formulas are, in reality, fifth-order formulas. This is true because the terms of $Y-y$ containing $h^{5}$, whose coefficients are not zero, also contain either $e^{\prime}, e^{\prime \prime}$, or $e^{\prime \prime \prime}$ as a factor.

To illustrate these facts, set $e^{\prime}=e^{\prime \prime \prime}=0, e^{\prime \prime}=1 / 1200 n, a_{1}=1 / 1000 n, a_{2}=\frac{3}{10}$, and $a_{3}=\frac{3}{4}$. These values lead to the one parameter set of fifth-order formulas (provided $n$ is large enough) given in the array below.

$$
\begin{array}{ll}
\frac{1}{1000 n} & \frac{1}{1000 n} \\
\frac{3}{10} & \frac{1}{10}[(-450 n+3)+450 n] \\
\frac{3}{4} & \frac{1}{8}[(2250 n-9)-2250 n+15] \\
1 & \frac{1}{81}[(-103500 n+459)+103500 n-490+112] \\
& \frac{1}{113}(105+0+500+448+81) .
\end{array}
$$

For the array (13), thirteen of the fifteen conditions are satisfied. The other two are approximately satisfied as shown below.

$$
\begin{aligned}
& 1-60 c^{\prime \prime} a^{2}=e^{\prime \prime}, \\
& 1-15 c a^{2} b a^{2}=-\frac{1}{4} e^{\prime \prime} .
\end{aligned}
$$

To be more specific, the array (13) gives a fifth-order formula with five evaluations if we choose $1200 \mathrm{nh}^{2}>1$ (smaller values of $n$ would suffice). 
9. Efficient Formulas of Various Orders. Using the principles already explained, we have derived other formulas. To avoid going into details and to conserve space, we will simply list a spectrum of such formulas, including formulas of the seventhand eighth-orders.

$$
\text { Formula (4-4) }
$$

$$
\begin{array}{cl}
\frac{1}{100} & \frac{1}{100} \\
\frac{3}{5} & \frac{1}{245}(-4278+4425) \\
1 & \frac{1}{8791}(524746-532125+16170) \\
\frac{1}{70092}(-179124+200000+40425+8791)
\end{array}
$$

Formula (5-5)

$$
\begin{array}{ll}
\frac{1}{900} & \frac{1}{900} \\
\frac{3}{10} & \frac{1}{10}(-4047+4050) \\
\frac{3}{4} & \frac{1}{8}(20241-20250+15) \\
1 & \frac{1}{81}(-931041+931500-490+112) \\
& \frac{1}{134}(105+0+500+448+81) \\
& \text { Formula }(6-6)
\end{array}
$$

$$
\begin{array}{ll}
\frac{1}{300} & \frac{1}{300} \\
\frac{1}{5} & \frac{1}{5}(-29+30) \\
\frac{3}{5} & \frac{1}{5}(323-330+10) \\
\frac{1}{15} & \frac{1}{810}(-510104+521640-12705+1925) \\
1 & \frac{1}{77}(-417923+427350-10605+1309-54) \\
& \frac{1}{3696}(198+0+1225+1540+810-77) \\
& \text { Formula }(7-7)
\end{array}
$$

$\frac{1}{192} \quad \frac{1}{192}$

$\frac{1}{6} \quad \frac{1}{6}(-15+16)$

$\frac{1}{2} \quad \frac{1}{186}(4867-5072+298)$

(17) $1 \frac{1}{31}(-19995+20896-1025+155)$

$\frac{5}{6} \quad \frac{1}{5022}(-469805+490960-22736+5580+186)$

$1 \frac{1}{2604}(914314-955136+47983-6510-558+2511)$

$$
\frac{1}{300}(14+0+81+110+0+81+14)
$$


(18)

$$
\begin{aligned}
& \frac{2}{9} \quad \frac{2}{9} \\
& \text { Formula (7-9) } \\
& \frac{1}{3} \frac{1}{12}(1+3) \\
& \frac{1}{2} \frac{1}{8}(1+0+3) \\
& \frac{1}{6} \frac{1}{216}(23+0+21-8) \\
& \frac{8}{9} \quad \frac{1}{729}(-4136+0-13584+5264+13104) \\
& \frac{1}{9} \frac{1}{151632}(105131+0+302016-107744-284256+1701) \\
& \frac{5}{6} \quad \frac{1}{13752 \bar{\sigma}}(-775229+0-2770950+1735136 \\
& +2547216+81891+328536) \\
& 1 \frac{1}{251888}(23569+0-122304-20384+695520 \\
& -99873-466560+241920) \\
& \frac{1}{2140320}(110201+0+0+767936+635040-59049 \\
& -59049+635040+110201) \\
& \text { Formula (8-10) } \\
& \frac{2}{9} \quad \frac{1}{18}(1+3) \\
& \frac{1}{3} \quad \frac{1}{12}(1+0+3) \\
& \frac{1}{2} \quad \frac{1}{8}(1+0+0+3) \\
& \frac{2}{3} \quad \frac{1}{54}(13+0-27+42+8) \\
& \frac{1}{6} \quad \frac{1}{432} \sigma(389+0-54+966-824+243) \\
& 1 \frac{1}{20}(-231+0+81-1164+656-122+800) \\
& \frac{5}{6} \quad \frac{1}{2} \overline{8}(-127+0+18-678+456-9+576+4) \\
& 1 \frac{1}{82} \bar{\sigma}(1481+0-81+7104-3376+72-5040-60+720) \\
& \frac{1}{840}(41+0+0+27+272+27+216+0+216+41) \\
& \frac{1}{9} \quad \frac{1}{9} \\
& \frac{1}{6} \quad \frac{1}{24}(1+3) \\
& \frac{1}{4} \quad \frac{1}{16}(1+0+3) \\
& \frac{1}{10} \quad \frac{1}{300}(29+0+33-12) \\
& \frac{1}{6} \quad \frac{1}{7} \frac{1}{2}(33+0+0+4+125) \\
& \frac{1}{2} \quad \frac{1}{36}(-21+0+0+76+125-162) \\
& \frac{2}{3} \quad \frac{1}{243}(-30+0+0-32+125+0+99) \\
& \frac{1}{3} \quad \frac{1}{324}(1175+0+0-3456-6250+8424+242-27) \\
& \frac{5}{6} \quad \frac{1}{324}(293+0+0-852-1375+1836-118+162+324) \\
& \frac{5}{6} \quad \frac{1}{162} \sigma(1303+0+0-4260-6875+9990+1030+0+0+162) \\
& 1 \frac{1}{4428}(-8595+0+0+30720+48750-66096+378 \\
& -729-1944-1296+3240) \\
& \frac{1}{84} \overline{0}(41+0+0+0+0+216+272+27+27+36+180+41)
\end{aligned}
$$

(20) 
Formulas (14), (18), and (20) have the orders indicated in the traditional sense while formulas (15), (16), (17), and (19) have the orders indicated from the new viewpoint and only for certain values of $h$.

10. Comparative Numerical Data. The comparative data given next was programmed in double precision on the IBM 7072 at Vanderbilt University. The differential equations used were chosen to give a spectrum of possible behavior so that any bias for a particular formula might be minimized. Time is given in minutes.

(1). The equation used was $y^{\prime}=y$ with the initial value $(1,0)$ and the interval of solution $x=0$ to $x=18$. The exact solution is $y=e^{x}$.

\begin{tabular}{|c|c|c|c|c|c|}
\hline Step-size & $\begin{array}{c}\text { Runge-Kutta } 4-4 \\
\text { Error }\end{array}$ & Time & Step-size & $\underset{\text { Error }}{\text { Formula }} 4-4$ & Time \\
\hline $\begin{array}{l}.04 \\
.06 \\
.09 \\
.12 \\
.15 \\
.18\end{array}$ & $\begin{array}{ll}.24 & 10^{2} \\
.12 & 10^{3} \\
.6010^{3} \\
.1810^{4} \\
.4410^{4} \\
.8910^{4}\end{array}$ & $\begin{array}{l}.63 \\
.42 \\
.28 \\
.21 \\
.17 \\
.15\end{array}$ & $\begin{array}{l}.04 \\
.06 \\
.09 \\
.12 \\
.15 \\
.18\end{array}$ & $\begin{array}{ll}.24 & 10^{2} \\
.12 & 10^{3} \\
.60 & 10^{3} \\
.18 & 10^{4} \\
.44 & 10^{4} \\
.89 & 10^{4}\end{array}$ & $\begin{array}{l}.63 \\
.42 \\
.28 \\
.22 \\
.17 \\
.15\end{array}$ \\
\hline Step-size & $\begin{array}{l}\text { Formula } 5-5 \\
\quad \text { Error }\end{array}$ & Time & Step-size & $\begin{array}{c}\text { Formula 6-6 } \\
\text { Error }\end{array}$ & Time \\
\hline $\begin{array}{l}.04 \\
.06 \\
.09 \\
.12 \\
.15 \\
.18\end{array}$ & $\begin{array}{ll}.16 & 10^{0} \\
.12 & 10^{1} \\
.90 & 10^{1} \\
.36 & 10^{2} \\
.11 & 10^{3} \\
.27 & 10^{3}\end{array}$ & $\begin{array}{l}.86 \\
.58 \\
.39 \\
.29 \\
.24 \\
.20\end{array}$ & $\begin{array}{l}.04 \\
.06 \\
.09 \\
.12 \\
.15 \\
.18\end{array}$ & $\begin{array}{ll}.94 & 10^{-3} \\
.10 & 10^{-1} \\
.12 & 10^{0} \\
.63 & 10^{0} \\
.23 & 10^{1} \\
.68 & 10^{1}\end{array}$ & $\begin{array}{r}1.12 \\
.75 \\
.50 \\
.38 \\
.31 \\
.26\end{array}$ \\
\hline Step-size & $\begin{array}{l}\text { Formula } 7-7 \\
\text { Error }\end{array}$ & Time & Step-size & $\begin{array}{c}\text { Formula 7-9 } \\
\text { Error }\end{array}$ & Time \\
\hline $\begin{array}{l}.04 \\
.06 \\
.09 \\
.12 \\
.15 \\
.18\end{array}$ & $\begin{array}{ll}.72 & 10^{-4} \\
.76 & 10^{-3} \\
.87 & 10^{-2} \\
.50 & 10^{-1} \\
.19 & 10^{0} \\
.59 & 10^{0}\end{array}$ & $\begin{array}{r}1.41 \\
.94 \\
.63 \\
.48 \\
.38 \\
.32\end{array}$ & $\begin{array}{l}.04 \\
.06 \\
.09 \\
.12 \\
.15 \\
.18\end{array}$ & $\begin{array}{ll}.13 & 10^{-4} \\
.79 & 10^{-4} \\
.12 & 10^{-2} \\
.88 & 10^{-2} \\
.41 & 10^{-1} \\
.14 & 10^{0}\end{array}$ & $\begin{array}{r}2.08 \\
1.39 \\
.93 \\
.70 \\
.56 \\
.47\end{array}$ \\
\hline Step-size & $\begin{array}{c}\text { Formula } 8-10 \\
\text { Error }\end{array}$ & Time & Step-size & $\begin{array}{c}\text { Formula } 8-12 \\
\text { Error }\end{array}$ & Time \\
\hline $\begin{array}{l}.04 \\
.06 \\
.09 \\
.12 \\
.15 \\
.18\end{array}$ & $\begin{array}{ll}.71 & 10^{-5} \\
.59 & 10^{-5} \\
.15 & 10^{-4} \\
.11 & 10^{-3} \\
.59 & 10^{-3} \\
.75 & 10^{-2}\end{array}$ & $\begin{array}{l}2.45 \\
1.64 \\
1.09 \\
.82 \\
.66 \\
.55\end{array}$ & $\begin{array}{l}.04 \\
.06 \\
.09 \\
.12 \\
.15 \\
.18\end{array}$ & $\begin{array}{ll}.74 & 10^{-5} \\
.43 & 10^{-5} \\
.33 & 10^{-4} \\
.36 & 10^{-3} \\
.21 & 10^{-2} \\
.86 & 10^{-2}\end{array}$ & $\begin{array}{r}3.28 \\
2.19 \\
1.46 \\
1.10 \\
.88 \\
.73\end{array}$ \\
\hline
\end{tabular}

The programming is such that time is not saved when entries in the formulas are zeroes. For example, due to the zeroes in the Runge-Kutta formula, the program could be designed to reduce the times given above slightly.

A study of the results will show the advantages of the higher order formulas. For example, the 8-10 formula takes .55 minutes and yields an error $.7510^{-2}$ while 
the Runge-Kutta formula takes .63 minutes and yields an error no better than $.2410^{2}$.

(2). The equation used was $y^{\prime \prime}=-\left(x y^{\prime}+y\right) /(x y)^{2}$ with initial value $(1,1,1)$ and the interval of solution $x=1$ to $x=19$. The exact solution is $y=\sqrt{1+2 \ln x}, y^{\prime}=1 / x y$.

Runge-Kutta 4-4

$\begin{array}{ccr}\text { Step-size } & \text { Error } & \text { Time } \\ .04 & .9610^{-5} & 1.51 \\ .06 & .4910^{-4} & 1.01 \\ .09 & .2510^{-3} & .68 \\ .12 & .7910^{-3} & .51 \\ .15 & .1910^{-2} & .41 \\ .18 & .3910^{-2} & .35\end{array}$

Formula 5-5

$\begin{array}{ccr}\text { Step-size } & \text { Error } & \text { Time } \\ .04 & .3810^{-7} & 2.04 \\ .06 & .2610^{-6} & 1.37 \\ .09 & .1610^{-5} & .91 \\ .12 & .4410^{-5} & .69 \\ .15 & .4910^{-5} & .55 \\ .18 & .1410^{-4} & .46\end{array}$

Formula 7-7

$\begin{array}{ccr}\text { Step-size } & \begin{array}{c}\text { Error } \\ .04\end{array} & \text { Time } \\ .06 & .4510^{-9} & 3.27 \\ .09 & .1410^{-8} & 2.18 \\ .12 & .9510^{-6} & 1.46 \\ .15 & .3610^{-5} & 1.10 \\ .18 & .9510^{-5} & .88 \\ & & \end{array}$

Formula 8-10

$\begin{array}{ccc}\text { Step-size } & \text { Frror } & \text { Time } \\ .04 & .1310^{-10} & 5.53 \\ .06 & .2110^{-9} & 3.69 \\ .09 & .3210^{-8} & 2.46 \\ .12 & .2110^{-7} & 1.85 \\ .15 & .8310^{-7} & 1.48 \\ .18 & .2510^{-6} & 1.24\end{array}$

Formula 4-4

$\begin{array}{ccr}\text { Step-size } & \text { Error } & \text { Time } \\ .04 & .3710^{-5} & 1.52 \\ .06 & .1910^{-4} & 1.02 \\ .09 & .9310^{-4} & .68 \\ .12 & .2910^{-3} & .51 \\ .15 & .7110^{-3} & .41 \\ .18 & .1510^{-2} & .35\end{array}$

Formula 6-6

$\begin{array}{ccr}\text { Step-size } & \text { Error } & \text { Time } \\ .04 & .1610^{-7} & 2.63 \\ .06 & .1910^{-6} & 1.76 \\ .09 & .2910^{-5} & 1.17 \\ .12 & .2310^{-4} & .88 \\ .15 & .1210^{-3} & .71 \\ .18 & .4910^{-3} & .59\end{array}$

Formula 7-9

$\begin{array}{ccc}\text { Step-size } & \text { Error } & \text { Time } \\ .04 & .1110^{-9} & 4.72 \\ .06 & .1710^{-8} & 3.15 \\ .09 & .2510^{-7} & 2.11 \\ .12 & .1510^{-6} & 1.58 \\ .15 & .4910^{-6} & 1.27 \\ .18 & .9910^{-6} & 1.06\end{array}$

(3). The equation used was $y^{\prime}=-2 x y^{2}$ with initial value $(1,0)$ and the in. terval of solution $x=0$ to $x=18$. The exact solution is $y=\left(1+x^{2}\right)^{-1}$.

$\begin{array}{ccr} & \text { Runge-Kutta } 4-4 & \\ \text { Step-size } & \text { Error } & \text { Time } \\ .04 & .7510^{-11} & .86 \\ .06 & .3910^{-10} & .58 \\ .09 & .2010^{-9} & .39 \\ .12 & .6410^{-9} & .29 \\ .15 & .1610^{-8} & .24 \\ .18 & .3310^{-8} & .20\end{array}$

$\begin{array}{ccr} & \text { Formula } 4-4 & \\ \text { Step-size } & \text { Error } & \text { Time } \\ .04 & .3310^{-11} & 1.15 \\ .06 & .1610^{-10} & .58 \\ .09 & .8410^{-10} & .39 \\ .12 & .2710^{-9} & .29 \\ .15 & .6410^{-9} & .20 \\ .18 & .1310^{-8} & .15\end{array}$


Formula 5-5

$\begin{array}{ccr}\text { Step-size } & \text { Error } & \text { Time } \\ .04 & .9410^{-14} & 1.15 \\ .06 & .6710^{-13} & .77 \\ .09 & .4810^{-12} & .52 \\ .12 & .1910^{-11} & .39 \\ .15 & .5710^{-11} & .31 \\ .18 & .1410^{-10} & .26\end{array}$

Formula 7-7

Step-size

.04

.06

.09

.12

.15

.18

Step-size
.04
.06
.09
.12
.15
.18

Formula 8-10 Error

$.5810^{-16}$

$.1010^{-16}$

$.4310^{-15}$

$.3410^{-14}$

$.1610^{-13}$

$.6010^{-13}$

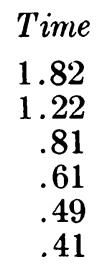

1.82

1.22

.81

.61

.41

Time
3.03
2.02
1.35
1.02
.81
.68

Step-size
.04
.06
.09
.12
.15
.18

Step-size
.04
.06
.09
.12
.15
.18

Formula 6-6 Error

Time

$.9710^{-15}$

$.1110^{-13}$

$.1410^{-12}$

$.1010^{-11}$

$.5110^{-11}$

$.2110^{-10}$

1.47

.98

.66

.50

.40

.33

Formula 7-9

Error

Time

$.4710^{-16}$

$.9910^{-16}$

$.2710^{-14}$

$.2410^{-13}$

$.1410^{-12}$

$.5710^{-12}$

2.60

1.74

1.16

.87

.70

.58

(4). The equation used was the same as in (2) above. The initial value was $(1,1,1)$ as above, but the interval now is $x=1$ to $x=181$. This data was programmed in extended double precision on an IBM 7094 at the Computation Division, Marshall Space Flight Center, Huntsville, Alabama.

$\begin{array}{ccr} & \text { Runge-Kutta } 4-4 & \\ \text { Step-size } & \text { Error } & \text { Time } \\ .04 & .1010^{-3} & 1.81 \\ .06 & .510^{-3} & 1.22 \\ .09 & .2610^{-2} & .81 \\ .12 & .8310^{-2} & .61 \\ .15 & .2010^{-1} & .50 \\ .18 & .4110^{-1} & .42\end{array}$

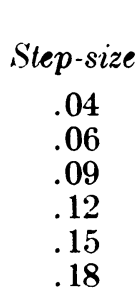

Formula 4-4

Formula 8-12

Error

Time

$.5710^{-16}$

3.97

$.37 \quad 10^{-16}$

2.65

$.9610^{-16}$

1.77

$.81 \quad 10^{-15}$

$.4910^{-14}$

$.2210^{-13}$

1.07

.89 


\begin{tabular}{cccccr} 
& Huť 6-8 & & \multicolumn{3}{c}{ Formula $6-6$} \\
Step-size & Error & Time & Step-size & Error & Time \\
.04 & $.4510^{-6}$ & 5.08 & .04 & $.9510^{-7}$ & 3.33 \\
.06 & $.5610^{-5}$ & 3.40 & .06 & $.8410^{-6}$ & 2.21 \\
.09 & $.7210^{-4}$, & 2.26 & .09 & $.6710^{-5}$ & 1.50 \\
.12 & $.4510^{-3}$ & 1.72 & .12 & $.2510^{-4}$ & 1.11 \\
.15 & $.1910^{-2}$ & 1.37 & .15 & $.5010^{-4}$ & .90 \\
.18 & $.5910^{-2}$ & 1.15 & .18 & $.3210^{-4}$ & .76
\end{tabular}

$\begin{array}{ccc} & \text { Formula } 7-7 & \\ \text { Step-size } & \text { Error } & \text { Time } \\ .04 & .2710^{-8} & 4.16 \\ .06 & .8910^{-7} & 2.78 \\ .09 & .1810^{-5} & 1.87 \\ .12 & .1110^{-4} & 1.40 \\ .15 & .4010^{-4} & 1.12 \\ .18 & .1010^{-3} & .95\end{array}$

Vanderbilt University

Nashville, Tennessee

1. A. HuŤa, "Une amélioration de la méthode de Runge-Kutta-Nyström pour la résolution numérique des équations différentielles du premier ordre," Acta Fac. Nat. Univ. Comenian. Math., v. 1, 1956, pp. 201-224. (Slovak and Russian summaries) MR 20 \#3635.

2. A. HUŤA, "Contribution à la formule de sixième ordre dans la méthode de RungeKutta-Nyström," Acta Fac. Nat. Univ. Comenian. Math., v. 2, 1957, pp. 21-24. (Slovak and Russian summaries) MR 20 \#3636.

3. W. KutTa, Z. Math. Phys., v. 46, 1901, pp. 435-453.

4. E. J. Nyström, "Ưber die numerische Integration von Differentialgleichungen," Acta Soc. Sci. Fenn., v. 50, 1925, No. 13, pp. 1-55.

5. C. Runge, Math. Ann., v. 46, 1895, pp. 167-178.

6. R. ZURMÜHL, "Runge-Kutta-Verfahren zur numerischen Integration von Differentialgleichungen $n$-ter Ordnung," $Z$. Angew. Math. Mech., v. 28, 1948, pp. 173-182. MR 10, 212. 